end-diastolic diameter was improved in 5 patients; right ventricular size and function improved in 3 patients. Three late deaths due to carcinoid progression occurred postoperatively at 39,5 , and 30 months.

\section{DISCUSSION}

Previous studies have shown that tricuspid and pulmonary valve replacement can improve symptoms and survival in patients with right heart failure due to carcinoid heart disease. ${ }^{1}$ Improved functional status and cardiac chamber size were also noted in our carcinoid patients with 4 valve replacements. Notably, a PFO was present in only 3 patients, and each was detected only by intraoperative transesophageal echocardiography with saline injection. Thus, our experience documents that an intracardiac shunt is not necessary for development of left-sided carcinoid valve disease, despite other reports. ${ }^{4,5}$

\section{CONCLUSIONS}

Although carcinoid heart disease with involvement of all 4 valves is rare, we conclude that quadruple valve replacement in symptomatic patients is a reasonable option that can result in functional improvement with acceptable risks.

\section{References}

1. Connolly HM, Schaff HV, Mullany CJ, Rubin J, Abel MD, Pellikka PA. Surgical management of left-sided carcinoid heart disease. Circulation. 2001;104(12 Suppl. 1):I36-40

2. Castillo JG, Filsoufi F, Rahmanian PB, Anyanwu A, Zacks JS, Warner RR, et al. Early and late results of valvular surgery for carcinoid heart disease. J Am Coll Cardiol. 2008;51:1507-9.

3. Gustafsson BI, Hauso O, Drozdov I, Kidd M, Modlin IM. Carcinoid heart disease. Int J Cardiol. 2008;129:318-24. Epub 2008 Jun 20.

4. Bhattacharyya S, Toumpanakis C, Caplin ME, Davar J. Analysis of 150 patients with carcinoid syndrome seen in a single year at one institution in the first decade of the twenty-first century. Am J Cardiol. 2008;101:378-81.

5. Mansencal N, Mitry E, Forissier JF, Martin F, Redheuil A, Lepere C, et al. Assessment of patent foramen ovale in carcinoid heart disease. Am Heart J. 2006;151: 1129.e1-6.

\title{
Successful resection of localized pulmonary mucormycosis after bone marrow transplantation using a virtual 3-dimensional pulmonary model on a personal computer
}

\author{
Hideyuki Maeda, MD, Masato Kanzaki, MD, Tamami Isaka, MD, PhD, and Takamasa Onuki, MD, PhD, \\ Tokyo, Japan
}

Mucormycosis is a rare and fatal infectious disease. Pulmonary mucormycosis most often occurs in patients with neutropenia, immune deficiencies, malignant disease, malnutrition, diabetes, and trauma, as well as in patients undergoing organ transplantation or chelation therapy involving deferoxamine. ${ }^{1,2}$ For control of the underlying disease, a polyene administration and surgical resection of infected lung segments may prove helpful. We report a case of localized pulmonary mucormycosis after bone marrow transplantation that was successfully resected using a virtual 3-dimensional pulmonary model on a personal computer.

\footnotetext{
From the Department of Surgery I, Tokyo Women's Medical University, Tokyo, Japan.

Disclosures: None

Received for publication April 30, 2010; accepted for publication May 11, 2010; available ahead of print June 11, 2010.

Address for reprints: Masato Kanzaki, MD, Department of Surgery I, Tokyo Women's Medical University, 8-1 Kawada-cho, Shinjuku-ku, Tokyo 162-8666, Japan (E-mail: kanzaki@chi.twmu.ac.jp).

J Thorac Cardiovasc Surg 2010;140:1434-6

$0022-5223 / \$ 36.00$

Copyright (c) 2010 by The American Association for Thoracic Surgery

doi:10.1016/j.jtcvs.2010.05.007
}

\section{CLINICAL SUMMARY}

A 28-year-old man, treated with allogenic bone marrow transplantation for acute lymphatic leukemia in March 2009, had progressive dyspnea and productive cough in May 2009. Chest radiography and computed tomography (CT) revealed a fungus ball in the left upper lobe of the lung (Figure 1, A). Mucor was detected by bronchoscopic biopsy. When liposomal amphotericin B (L-AMB) was administered, the size of the fungus ball reduced immediately (Figure 1,B). However, because the patient became resistant to L-AMB, he was referred to our division for surgery for pulmonary mucormycosis. Preoperative blood chemistry analysis showed pancytopenia and renal insufficiency (the serum creatinine level $1.94 \mathrm{mg} / \mathrm{dL}$ ) caused by ongoing L-AMB administration. Chest CT demonstrated an 18-mm cavitary mass in $\mathrm{S} 1+2 \mathrm{c}$ of the left lung. For preoperative simulation of surgical resection, we used free software application such as CTTRY, where we could mark on pulmonary arteries, veins, bronchi, and fungus ball of the images on high-resolution CT images. With 60 2-mm highresolution CT images of the fungus ball and hilum, in DICOM (Digital Imaging and Communications in Medicine) format, we obtained 3-dimensional images for a patient 

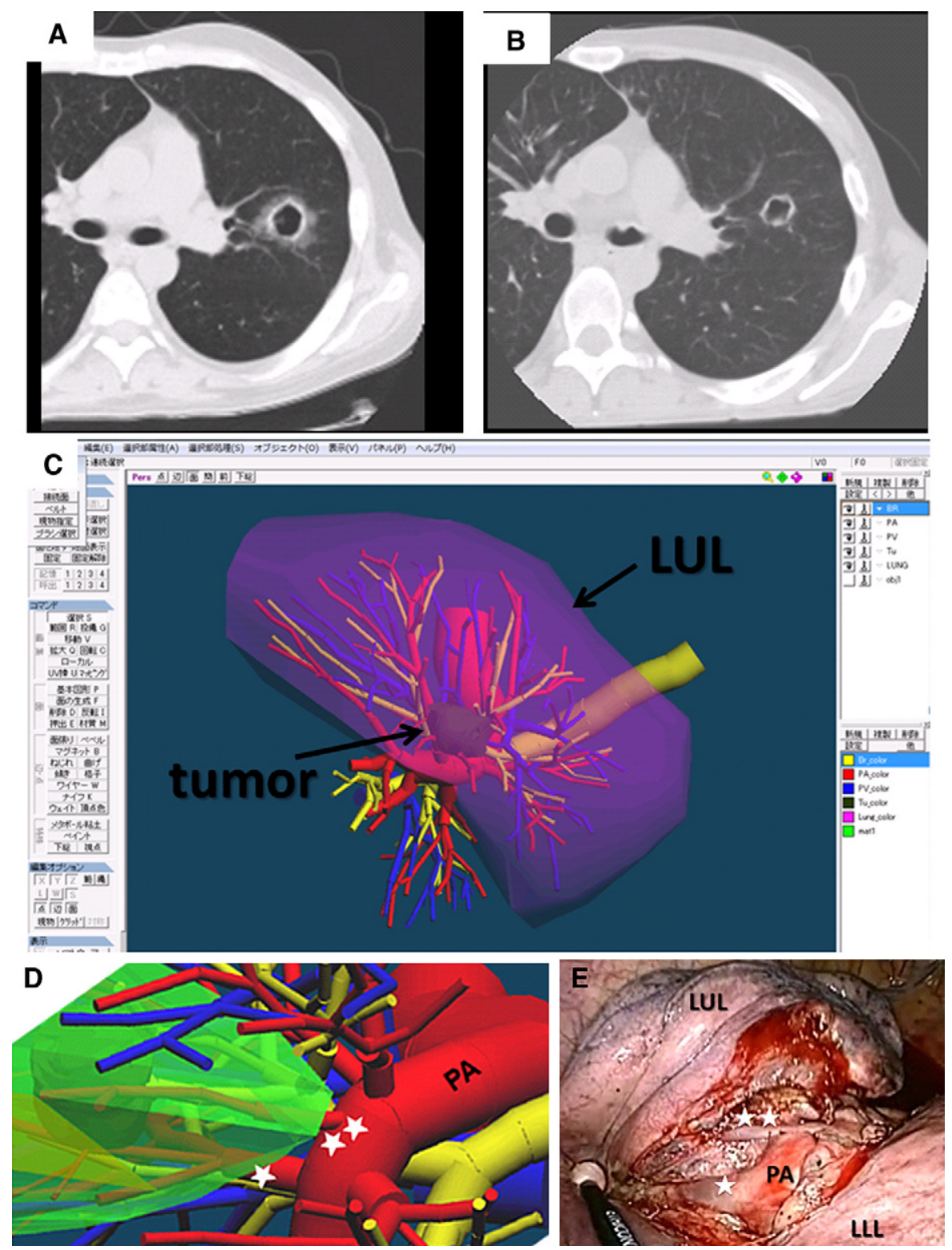

FIGURE 1. A, A computed tomogram (CT) shows a fungus ball in the $\mathrm{S} 1+2$ of the left upper lobe of the lung. B, The size of a fungus ball was decreased by administering liposomal amphotericin B (L-AMB) before the operation. C, Three-dimensional images of the left lung based on high-resolution CT with 2-mm CT slices. Pulmonary arteries, veins, and bronchi are shown in red, blue, and yellow. A fungus ball was located in $\mathrm{S} 1+2 \mathrm{of}$ the left lung. D and $\mathrm{E}$, Comparison of 3-dimensional images and intraoperative findings. $T$, Tumor; $P A$, interlobar pulmonary artery; $\star$, lingular segmental pulmonary artery (A4 $+5) ; \star \star$, apicoposterior segmental branch $(\mathrm{A} 1+2 \mathrm{c})$; $L U L$, left upper lobe; $L L L$, left lower lobe.

using Metasequoia shareware (Figure 1,C). We planned the operation based on the 3-dimensional image; 3 subsegments (ie, S1+2c, S3a, and S4a) needed to be resected to remove the fungus ball completely. Video-assisted thoracoscopic surgical resection of the subsegments was performed (Figure 1, $D$ and $E$ ). Macroscopic examination of the surgical specimen revealed a 20-mm left upper lobe fungus ball cavity (Figure 2, A). Microscopically, a Grocott stain highlighted the fungal structures (Figure 2, B). The patient's postoperative course was uneventful. He was discharged on postoperative day 9 . He is active without respiratory limitation.

\section{COMMENT}

Successful treatment of mucormycosis consists of early diagnosis, correction of underlying risk factors, performing surgical resection, and prompt antifungal therapy. It is difficult to diagnose pulmonary mucormycosis, because chest 


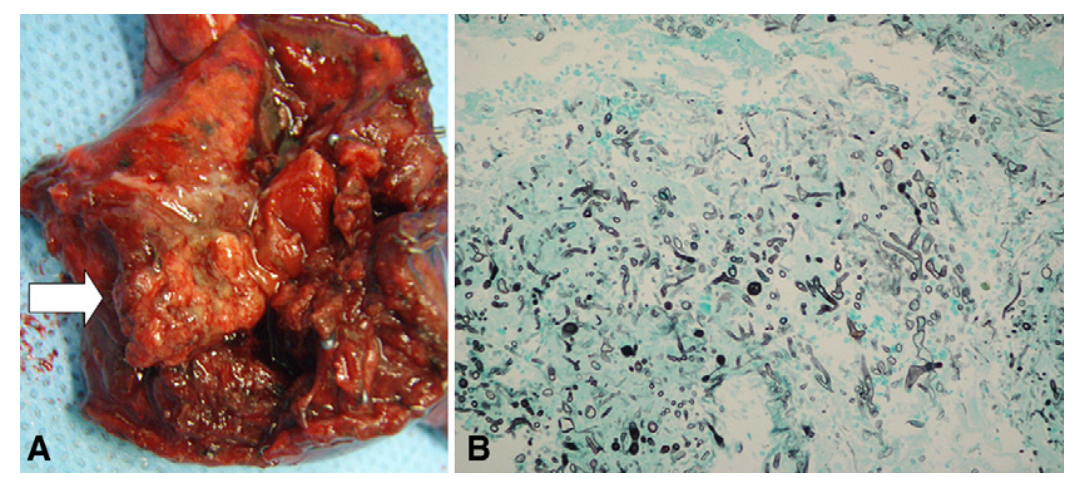

FIGURE 2. A, Macroscopic findings. The size of the mass is $20 \mathrm{~mm}$. A fungus ball is present inside the mass (white arrow). B, Photomicrograph of the lesion shows characteristic broad, aseptate hyphae with stubby, right-angled, fingerlike branches in a background of necrotic tissue (Grocott's methenamine silver stain, original magnification $\times 200$ ).

radiographic findings are variable, culture results are commonly negative, and there are currently no routinely available serum immunologic assays for it. Pulmonary mucormycosis is usually diagnosed by histopathology. Transbronchial biopsy procedures or a surgical lung biopsy procedure may be required to obtain an adequate specimen for diagnosis. Generally, a polyene, such as the AMB or L$\mathrm{AMB}$, is administered as primary antifungal therapy. However, blood vessel thrombosis and resulting tissue necrosis during mucormycosis can result in poor penetration of antifungal agents to the site of infection. Therefore, surgical debridement of necrotic tissues may be critical for complete eradication of mucormycosis. ${ }^{3}$ Roden and associates ${ }^{4}$ reported that the mortality rate of pulmonary mucormycosis is $76 \%$ (170/220 patients). However the patients who underwent surgical resection were significantly more likely to survive than those who did not. ${ }^{4}$

In this case, although the patient's pulmonary mucormycosis had been controlled and localized by administering L-AMB, surgical resection was selected. We prefer segmentectomy or more conservative resection to lobectomy for peripheral, small, stage I non-small cell lung cancer. Therefore, we performed multi-subsegmentectomy for the localized pulmonary mucormycosis. Because we constantly focus on the possibility of lung-saving procedures, we attempted to reconstruct using anatomically correct images with a freeware program. After the images had been uploaded to a personal computer, the pulmonary arteries, pulmonary veins, and bronchi were traced in the images. The location and thickness of the bronchi and pulmonary vasculature were rendered as different-sized cylinders. Next, on the basis of the resulting numerical data, 3-dimensional images were reconstructed. The reconstructed images can be manipulated by virtual surgical procedures such as reshaping, cutting, and moving. ${ }^{5}$

Although the incidence of mucormycosis is increasing, surgical excision of localized disease appears to be the most reliable method of treating pulmonary mucormycosis.

In conclusion, early detection and aggressive treatment for pulmonary mucormycosis offer the best chance of patient survival.

\section{References}

1. Pauls DR, Ravenel JG, Judson MA. A neutropenic patient with rapidly progressive lung lesion. Chest. 2004;126:1364-7.

2. Sharifipour F, Rezaeetalab F, Naghibi M. Pulmonary fungal infections in kidney transplant recipients: an 8-year study. Transplant Proc. 2009;41:1654-6.

3. Spellberg B, Walsh TJ, Kontoyiannis DP, Edwards J Jr, Ibrahim AS. Recent advances in the management of Mucormycosis: from bench to bedside. Clin Infect Dis. 2009;48:1743-51.

4. Roden MM, Zaoutis TE, Buchanan WL, Knudsen TA, Sarkisova TA, Schaufele RL, et al. Epidemiology and outcome of zygomycosis: a review of 929 reported cases. Clin Infect Dis. 2005;41:634-53.

5. Takamasa O. Virtual reality in video-assisted thoracoscopic lung surgery. Kyoubu Geka. 2009;62:733-8. 